\title{
Energy Efficiency in Wireless Sensor Network using Randomized Switching for Maximizing Lifetime
}

\author{
Vasudeva Pai, Karthik Pai B H, Udaya Kumar K Shenoy
}

\begin{abstract}
Wireless Sensor Network is distributed networks of sensors which have the ability to sense, process and communicate. Sensor nodes are also responsible for collection of data. Due to the limited battery power of sensor node energy consumption is an essential issue. To reduce the energy consumption balancing of node load is one of the major task. In this paper, we have used switching algorithm to switch the nodes to balance the node load which further increases the life time of each node by finding the shortest path to destination from the source node based on the threshold energy. Further we applied base localization algorithm to check the lifetime of each node.
\end{abstract}

Keywords: Load balancing, localization, MATLAB, Wireless Sensor Network

\section{INTRODUCTION}

Wireless Sensor Networks (WSN) are used in many of the application like vehicle monitoring, military, healthcare, forest monitoring, environmental, industrial biological and other commercial applications. Sensors in networks are the important concern in the field of sensor technology, which is composed of sensor nodes which has, embedded processors, one sensor, limited memory and are battery operated and capable of sensing, transmitting the collected information, which influences in variety of application. In WSN battery powered sensor nodes is a main component which having the low-cost and power. Lifetime of the network has to be managed by utilizing the limited energy resources to extend the life time of networks. Energy can be conserved by using popular three techniques they are, duty-cycling[1], aggregation of data[2], load balancing[4,5] Instead of first two techniques load balancing technique will organize network topology to have uniform load on node while forwarding a data. Node nearer to sink will run out of energy due to higher data to be send. Therefore load balancing technique is suitable for maximizing the life time of network.

Revised Manuscript Received on October 30, 2019.

* Correspondence Author

Vasudeva Pai*, department of Information Science \& Engineering, NMAM Institute of Technology, Nitte, Karnataka, India.

Karthik Pai B H, department of Information Science \& Engineering, NMAM Institute of Technology, Nitte, Karnataka, India.

Udaya Kumar K Shenoy, department of Information Science \& Engineering, NMAM Institute of Technology, Nitte, Karnataka, India.

(C) The Authors. Published by Blue Eyes Intelligence Engineering and Sciences Publication (BEIESP). This is an open access article under the CC BY-NC-ND license (http://creativecommons.org/licenses/by-nc-nd/4.0/)

\section{Localization}

Localization in WSN used to identify the current location of the sensor nodes. There are two techniques in it they are Centralized Localization and Distributed Localization

In Centralized localization all the measurements of node will be gathered at the base station.

In Distributed localization sensor nodes will calculate the measurement themselves and then communicate with other node, distributed localization can be divided to two parts, Range based and Range free localization. In range based localization, nodes will calculate the distance from one node to another node. In range free localization, node will not estimate point-to-point distance between the nodes.

\section{Matlab}

It is a high performance language for technical computing. An interactive system where array is a data element of Matlab which does not require dimensioning and it supports $\mathrm{C}, \mathrm{C}++$, java, and c \#python.

\section{LITERATURE REVIEW}

S K Kajal Arefin Imon et al.[6] proposed an efficient randomized switching algorithm (RaSMaLai) to maximize the lifetime of the network by switching the node path to balance the load on each node based on the bounded balanced tree concept.

Mao Ye et al.[7] suggested energy efficiency methods importance to prolong the lifetime of network. Scalability of network and its lifetime in wireless network can be improved by Clustering, the EECS schema of clustering helps in gathering timely data over a period of time. With this cluster EECS approach the load, can be equally distributed among cluster heads by utilising the residual energy in the form of radio communication which are present in local.

A Jeong Jeong et al. [8] proposed with a routing protocol known by MPDA that would to increase precision of measurement and benefit in energy saving in Wireless sensor network. As per each cluster characteristic, MPDA decides on aggregation period. This eventually impacted to save energy and also map the measurement accurately. Performance evaluation of MPD is done, by comparing MPDA alongside LEACH in MATLAB simulation. The Alive nodes and intrinsic difference of sensed and real valuation is observed in evaluation metric in the experiment conducted for analysis. MPDA is fund better than LEACH as fewer errors and lower energy consumed is recorded. 
Md. Taybur Rahama et al.[9] worked on reduction in delay time of data, by using efficient protocol for routing. The New Protocol designed, has a new $\mathrm{CH}$ which will be selected by existing $\mathrm{CH}$ rather than the BS which was based on unused energy and node distance, where $\mathrm{CH}$ is minimum of all the nodes present in that cluster. Not being similar to Modified Leach (MLEACH), newly proposed protocol is occurring in terms of leftover energy of current $\mathrm{CHs}$ lower than a value which is basically threshold one. The New protocol has a advantage that formation time of cluster and failure of network will be lesser. It is better than MLEACH, as protocol proposed has better network fault tolerance and good ratio of packet delivery.

Seung-Wan Han et al. [10] proposed two algorithms named by Branch \& Bound and annealing algorithm, The B\&B mainly for wireless sensor network with small scale operations, whereas simulated annealing algorithm for higher scale. MWST, Minimum Wireless Spanning Tress is outperformed by MST, Minimum Spanning Tree. For optimising the energy usage and eliminate packet delay to some extent, one of representative tree is used for sensor networks in almost all cases.

Hong Luo et al. [11] proposed Adaptive Fusion Steiner Tree (AFST), this optimizes the costs of both data fusion and data transmission along with route information.

\section{OBJECTIVE OF THE PROPOSED WORK}

The main objective of the proposed work is to optimize the energy efficiency by Randomized Switching for maximizing the life time algorithm

- Balancing load of node by switching the one node to another node.

- Analysing the impact of threshold bond on life time of network.

- Comparing the life time of node in different scenario.

- Determining the average mean square error during localization

\section{METHODOLOGY}
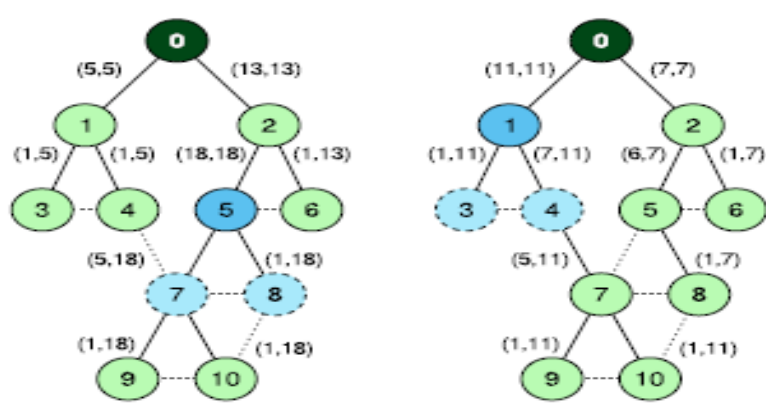

Fig.1 switching of nodes from one node to another node.

Switching algorithm starts by initializing the node load and path load, set the switch count to zero and switching probability to half and select the node with highest node load, switching process continues until some nodes are selected for maximum switch count. Once the switch count reaches to maximum then update the tree. Check for the threshold bonded condition every time if it satisfies the tree is updated again, otherwise insert child node to queue and update the switch count and check queue until it becomes empty. At every step node is removed from the queue in FIFO order and a list is removed with potential parent. If that removed child node has not any potential parent then child nodes are added to the queue and considered in switch in the next round, if child node has potential parent then switching decision has to be taken. Switching can be done by selecting each node from list to be a new parent, if there is no switching decision needed taken then current parent and its child are added to the queue for the next consideration. Switching of node will balance the tree by which the life time of sensor node will increase.

Further apply base localization algorithm which is a range free localization to check the sink location so that sending of data from each node to sink node will be efficient.

\section{RESULT AND DISCUSSION}

This section discusses impact of threshold bond on life time, and life time of sensors at runtime, compares the life time of node in two scenarios and average mean square error during localization.

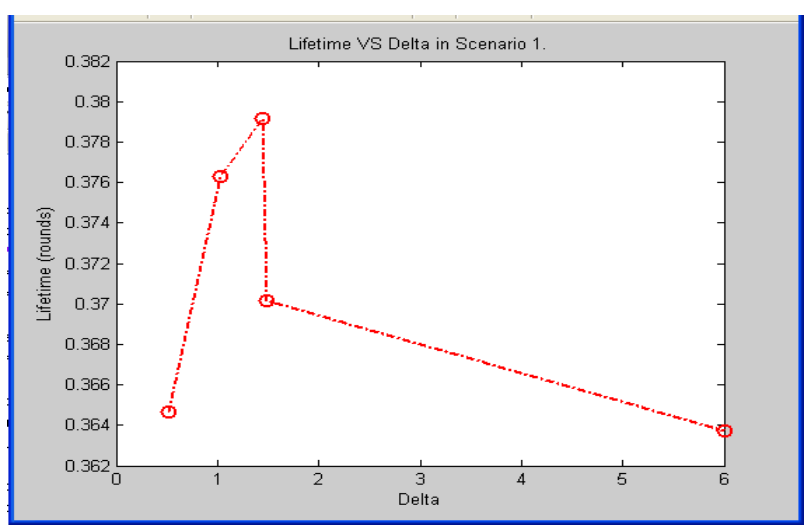

Fig.2 Impact of delta on lifetime of sensors

Fig.2 indicates the impact of delta on lifetime of sensors, we can observe that as the delta decreases, tree with higher life time is obtained.

Table 1 Impact Of Threshold Bond (Delta) On Life Time

\begin{tabular}{|c|c|}
\hline Delta value & Life time of network \\
\hline 0.5 & 0.3647 \\
\hline 1 & 0.3794 \\
\hline 1.5 & 0.3762 \\
\hline 2 & 0.3704 \\
\hline 6 & 0.3638 \\
\hline
\end{tabular}

In the Table 1, we can observe that in certain condition lifetime of node increases and then decreases this is because impact of threshold bond, but we can say that the life time of sensors can be increases when delta value decreases. 


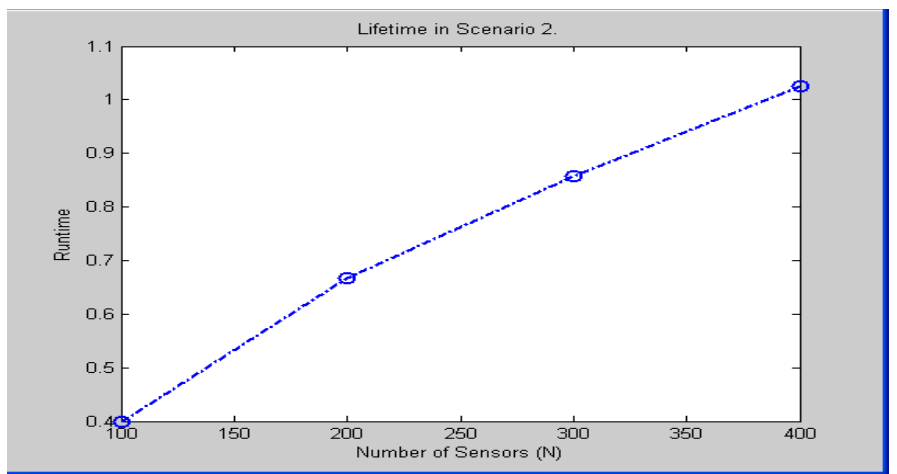

Fig 3. .Life time of sensors at run time.

When sink node is deployed in one side of the deployment area, run time of network increases with increasing of network size.

Table 2 Life time of sensors at run time

\begin{tabular}{|c|c|}
\hline Number of nodes & Life time of node \\
\hline 100 & 0.4382 \\
\hline 200 & 0.6541 \\
\hline 300 & 0.8695 \\
\hline 400 & 1.2365 \\
\hline
\end{tabular}

As the network size that is number of node increases the run time of network is also increases.

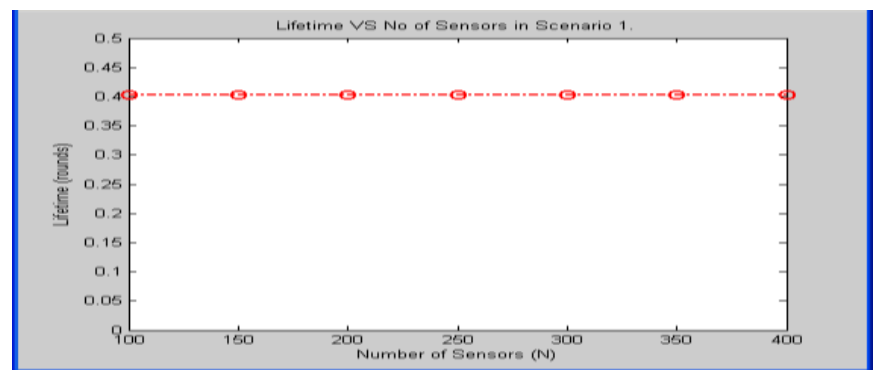

Fig.4 Life Time Of Sensors In Scenario1 When Sink Node Is Placed At Middle Of The Deployment Area.

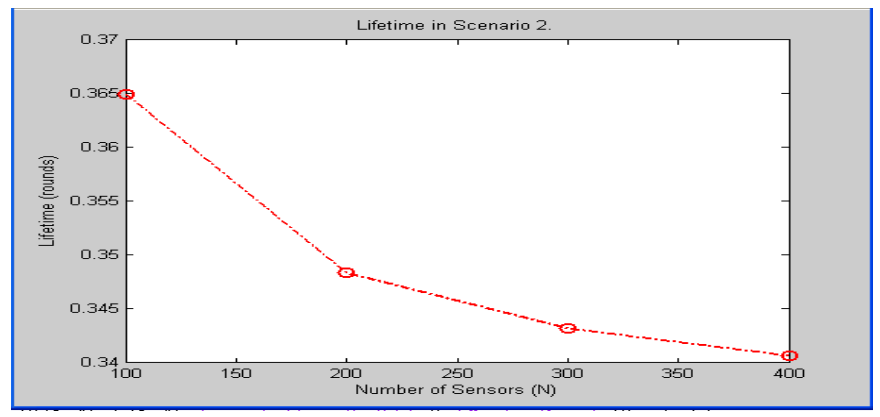

Fig.5 Life time of sensors in scenario2 when the sink is placed at one side of the deployment area.

Table 3 Comparison Of Life Time Of Network In Two Scenarios

\begin{tabular}{|c|c|c|}
\hline $\begin{array}{c}\text { Number of } \\
\text { nodes }\end{array}$ & $\begin{array}{c}\text { Life time in } \\
\text { Scenario 1 }\end{array}$ & $\begin{array}{c}\text { Life time in Scenario } \\
\mathbf{2}\end{array}$ \\
\hline 100 & 0.4437 & 0.3649 \\
\hline 200 & 0.3998 & 0.3484 \\
\hline 300 & 0.3810 & 0.3432 \\
\hline 400 & 0.3703 & 0.3407 \\
\hline
\end{tabular}

In Table 3 we can observe two scenarios, scenario 1 indicates when the sink node is deployed in the centre of the deployment area $(50 \mathrm{~m}, 50 \mathrm{~m})$. Scenario 2 indicates when the sink node is deployed in one side of the deployment area $(100 \mathrm{~m}, 50 \mathrm{~m})$. In scenario 1 we can say that the life time of sensors are balanced by constant decrease of life tie but in the scenario 2 we can see the sudden decrease of life time of sensors.

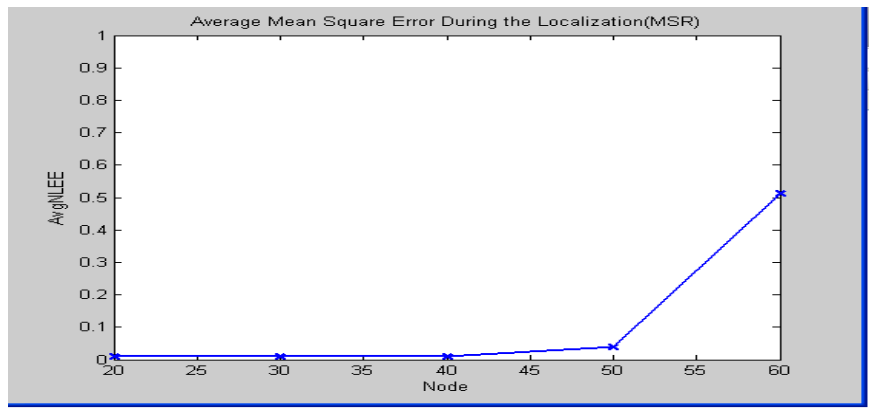

Fig.6 Average Mean Square Error During Localization

Life time of node increases due to localized base station by which the identifying of node becomes easier with less consumption of energy.

Table 4 Average Mean Square Error During Localization

\begin{tabular}{|c|c|}
\hline Number of nodes & Average mean square error \\
\hline 20 & 0.0357 \\
\hline 30 & 0,0698 \\
\hline 40 & 0.0854 \\
\hline 50 & 0.0956 \\
\hline 60 & 0.5249 \\
\hline
\end{tabular}

Table 4 indicates the average mean square error of node when base localization algorithm is applied to the scenario 2 . Average mean square error nothing but the difference between estimated values and what is estimated. Here we can observe that the average mean square value increases as the node increases which tells that life time of network increases when the location of node is known.

\section{CONCLUSION}

The proposed work consists, comparison of life time of network in two scenarios, and impact of threshold bond on life time of nodes. By this we can say that life time of network increases when the node load is balanced. Further we applied base localization algorithm to utilize efficient energy by providing the location of sink to all the nodes.In future we need to discover the sink's consistent incorporated localization. We need to locate a sensible and point by point quantitative correlation of existing range-free algorithms to decide the framework designs under which each is enhanced.

\section{REFERENCES}

1. F.J Wu and Y.C Tseng, "Distributed wake-up scheduling for data collection in tree based wireless sensor networks", Comm.Letters, vol.13, pp.850-852, November 2009.

2. G. Anastasi, M.Conti, M.Di Francesco, and A.Passarella, "Energy conversation in wireless sensor network: A survey", AdHoc Netw., vol.7, pp.537-568, May 2009.

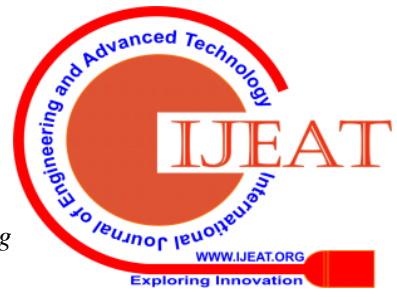


3. D.Kumar, T.C.Aseri, and R.B. Patel, "EECDA:Energy efficient clustering and data aggregation protocol for heterogeneous wireless sensor network", Communication, vol.VI,no. 1, pp 113-124,2011.

4. S. Toumpis and S.Gitzenis, "Load balancing in wireless sensor networks using Kirchhoff's voltage law", in IEEE INFOCOM, 2009,pp. 1656-1664.

5. J. Liang, J.Wang, J.Cao, J.Chen and M.Lu, “An efficient algorithm for constructing maximum lifetime tree for data gathering without aggregation in wireless sensor network", in INFOCOM, 2010,pp.1-5.

6. SK Kajal Arefin Imon, Adnan Khan, Mario Di Francesco, Sajal K.Das, "RaSMaLai:A Randomized Switching Algorithm for Maximizing Lifetime in Tree-based Wireless Sensor Networks", 2013 IEEE

7. .Mao Ye, Guihai Chen, Jie Wu, "An Energy Efficient Clustering Scheme in Wireless Sensor Networks", 2005 IEEE

8. A-JeongJeong,Se-Mi Kim,Chae-Seok Lee, and Jong-Deok Kim, "Multi-Periodic Data Aggregation Scheme Based on Interest for Energy Efficiency in Wireless Sensor Network", $20129^{\text {th }}$ International Conference on Ubiquitous Intelligence and Computing and $9^{\text {th }}$ International Conference on Autonomic and Trusted Computing.

9. Md. Taybur Rahama, Monir Hossen, and Md. Muminur Rahman, "A Routing Protocol for Improving Eneregy Efficiency in Wireless Sensor Networks", 2016 IEEE.

10. Seung-Wan Han, In-SeonJeong, Seung-HoKang, "Low latency and energy efficient routing tree for wireless sensor networks with multiple mobile sinks", Journal of Network and Computer Application 36(2013) 156-166.

11. Hong Luo, Jun Luo, Yonghe Liu, Sajal K. Das, “Adaptive Data Fusion for Energy Efficient Routin in Wireless Sensor Networks", IEEE TRANSACTIONS ON COMPTERS, VOL. 55, NO. 10, OCTOBER 2006.

12. Wook Choi, GlacomoGhidni and Sajal K. Das, "A Novel Framework for Energy-Efficient Data Gathering with Random Coverage in Wireless Sensor Networks", ACM Transactions on Sensor Networks, Vol.8, No.4 Article 36, Publication date: September 2012.

13. K. Kalaiselvi, G.R. Suresh, "Improved clustering protocol for eergy efficiency algorithms in wireless sensor networks", IEEE-31661.

14. Suhail Ahamed Nisar Shaha, Vasudeva Pai, Udaya Kumar K Shenoy, "Comparison of wireless routing protocols over FTP traffic in mobile and non-mobile nodes", 2017 ICICICT, pp, 349-353

\section{AUTHORS PROFILE}

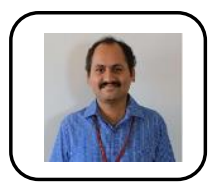

Vasudeva Pai received the B.E. and M.Tech degrees from Visvesvaraya Technological University, Belagavi, India, in 2005 and 2010, respectively, both in Computer Science Engineering. $\mathrm{He}$ is currently working as Assistant Professor in NMAM Institute of Technology, Nitte, India. His research interests include Wireless Sensor Networks, mobility management, Cryptography and Network Security, SDN, and IoT

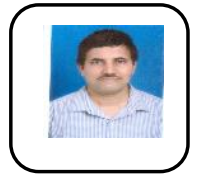

Karthik Pai B H received the B.E. degree(1998) from AEC, Bhatkal and M.Tech, Ph.D degrees from Visvesvaraya Technological University, Belagavi, India, in 2003 and 2018, respectively, both in Computer Science Engineering. He is currently working as Professor in NMAM Institute of Technology, Nitte, India. His research interests include Mobile Ad Hoc Networks, Software Engineering, Cryptography and Network Security, Design Thinking, and IoT.

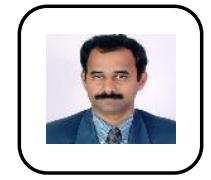

Udaya Kumar K Shenoy recevied the bachelor's degree (1992) in computer science, Master's degrees (200) in computer application from Mangalore University and Ph.D. (2009) from National Institute of Technology Karnataka, Surathkal. He is currently working as Professor in NMAM Institute of Technology, Nitte, India. His research interests include Wireless Networks, Optimization, Multimedia communication and Network Security 